\title{
Poststroke recovery: a frontier for intervention trials
}

\author{
Michelle P. Lin \\ Department of Neurology, Mayo Clinic, Jacksonville, Florida, United States
}

Key words: selective serotonin reuptake inhibitor, poststroke, recovery

(Neurol Neurochir Pol 2020; 54 (6): 488-489)

Acute stroke therapy and stroke prevention have markedly changed the landscape of stroke practice. Nonetheless, stroke affects nearly 800,000 individuals annually, and more than two thirds of stroke survivors receive rehabilitation. Unfortunately, there is no effective therapy for stroke rehabilitation and recovery. Early swallowing evaluation has been shown to improve poststroke dysphagia. In 2019, the PJNNS published the results of a randomised controlled trial showing that comprehensive therapy for dysphagia is effective and can reduce serious complications of swallowing disorders in patients who have experienced ischaemic stroke [1].

As for pharmacotherapeutic options for stroke recovery, selective serotonin reuptake inhibitors (SSRIs) such as fluoxetine are now commonly prescribed after stroke due to the numerous positive effects of SSRIs on mood and language recovery. In 2011, FLAME, a double-blind, placebo-controlled trial comparing fluoxetine $20 \mathrm{mg}$ daily vs placebo for three months after acute ischaemic stroke showed that fluoxetine was associated with better motor recovery [2]. Despite its small sample size of less than 60 patients on each arm, the FLAME trial aroused great interest among researchers given the absence of effective therapy for stroke recovery. The need for poststroke recovery therapeutics led to a collaborative design with the same basic protocol covering three larger trials conducted in the UK, Sweden, Australia, New Zealand and Vietnam of fluoxetine for poststroke recovery (FOCUS [3], AFFINITY [4], EFFECT [5]), to assess whether fluoxetine after acute stroke can improve functional outcomes. These studies, published in 2019 and 2020, wholeheartedly demonstrated that fluoxetine failed to improve functional outcome six months after acute stroke, and actually increased the risk of falls and bone fracture [3-5]. Nevertheless, fluoxetine did reduce the occurrence of depression, which is what SSRIs are designed to do. Individual-level meta-analysis of these three trials is underway.

In this issue of PJNNS, Bembenek et al. [6] have conducted a double-blind, randomised placebo-controlled trial (FOCUS) to evaluate the effects of fluoxetine $20 \mathrm{mg}$ daily versus placebo for six months after acute stroke. This similarly shows that fluoxetine for six months does not improve motor recovery or functional outcome six and 12 months poststroke in a Polish cohort. The authors similarly show that those on fluoxetine are less likely to require additional antidepressant medications.

The FOCUS trial has strengths. Firstly, it is evaluating a Polish cohort that has not been previously studied with the same rigour and clinical trial design. Secondly, the investigators have used various general and focused outcome scales to thoroughly assess the impact of fluoxetine on stroke recovery. These scales include the modified Rankin scale (mRS), Barthel index, and National Institutes of Health Stroke Scale (NIHSS) for general stroke outcome, MRC and Brunnstrome scale for hand motor function, as well as stroke impact scale (SIS) and mental health inventory (MHI) for health status and mood assessment, visual analogue scale (VAS) and EuroQoL-5 Dimensions-5 Levels (EQ5D-5L) for health-related quality of life, which are all important functional domains in stroke recovery.

The main limitation of FOCUS is its small sample size from a single centre in Poland. Furthermore, the study included patients with ischaemic stroke or intracranial haemorrhage, unlike FLAME that only included patients with ischaemic stroke. SSRIs are associated with poor outcomes in patients with intracranial haemorrhage, so the inclusion of both ischaemic and haemorrhagic stroke 
could have attenuated the potential benefit of fluoxetine. The interaction of SSRIs with the metabolism of clopidogrel and proton-pump inhibitors is well understood, such that co-administration of fluoxetine and a P2Y12 inhibitor may further influence the clinical outcome of recurrent stroke, haemorrhage and other bleeding complications. The planned individual-level meta-analysis of FOCUS, AFFINITY, and EFFECTS will provide more insight into the effects of fluoxetine in specific patient populations and with specific background therapies.

There is a growing body of high-level evidence failing to demonstrate a benefit of SSRIs in poststroke motor recovery. With ageing populations, and the concomitant increasing prevalence of stroke, the search for safe and effective therapies to improve stroke recovery in motor, cognition, and language functions, as well as mood and quality of life, represent the next frontier in stroke research.

Funding: National Centre for Advancing Translational Sciences of the National Institutes of Health, under Award Number KL2TR002379.

Acknowledgement: Richard and Mary Fink Family Foundation. Disclosures: None.

\section{References:}

1. Krajczy E, Krajczy M, Luniewski J, et al. Assessment of the effects of dysphagia therapy in patients in the early post-stroke period: a randomised controlled trial. Neurol Neurochir Pol. 2019; 53(6): 428-434, doi: 10.5603/PJNNS.a2019.0053, indexed in Pubmed: 31724147.

2. Chollet F, Tardy J, Albucher JF, et al. Fluoxetine for motor recovery after acute ischaemic stroke (FLAME): a randomised placebo-controlled trial. Lancet Neurol. 2011; 10(2): 123-130, doi: 10.1016/S14744422(10)70314-8, indexed in Pubmed: 21216670.

3. FOCUS Trial Collaboration. Effects of fluoxetine on functional outcomes after acute stroke (FOCUS): a pragmatic, double-blind, randomised, controlled trial. Lancet. 2019; 393(10168): 265-274, doi: 10.1016/S0140-6736(18)32823-X, indexed in Pubmed: 30528472.

4. AFFINITY Trial Collaboration. Safety and efficacy of fluoxetine on functional outcome after acute stroke (AFFINITY): a randomised, double-blind, placebo-controlled trial. Lancet Neurol. 2020; 19(8): 651-660, doi: 10.1016/S1474-4422(20)30207-6, indexed in Pubmed: 32702334.

5. EFFECTS Trial Collaboration. Safety and efficacy of fluoxetine on functional recovery after acute stroke (EFFECTS): a randomised, double-blind, placebo-controlled trial. Lancet Neurol. 2020; 19(8): 661-669, doi: 10.1016/S1474-4422(20)30219-2, indexed in Pubmed: 32702335.

6. Bembenek J, Niewada M, Klysz B, et al. Fluoxetine for stroke recovery improvement: the double-blind, randomized placebo-controlled FOCUS-Poland trail. Neurol Neurochir Pol. 2020; 54(6): 544-551, doi: 10.5603/PJNNS.a2020.0099. 